\title{
L'Église canadienne à partir de la formation de la province ecclésiastique de Québec (1844) jusqu'au cinquième Concile provincial de Québec (1873). Évolution des structures et mentalité des évêques.
}

\section{Jacques Grisé}

Volume 39, 1972

URI : https://id.erudit.org/iderudit/1007255ar

DOI : https://doi.org/10.7202/1007255ar

Aller au sommaire du numéro

\section{Éditeur(s)}

Les Éditions Historia Ecclesiæ Catholicæ Canadensis Inc.

\section{ISSN}

0318-6172 (imprimé)

1927-7067 (numérique)

Découvrir la revue

Citer cet article

Grisé, J. (1972). L’Église canadienne à partir de la formation de la province ecclésiastique de Québec (1844) jusqu'au cinquième Concile provincial de Québec (1873). Évolution des structures et mentalité des évêques. Sessions d'étude - Société canadienne d'histoire de l'Église catholique, 39, 9-28. https://doi.org/10.7202/1007255ar

Tous droits réservés @ Les Éditions Historia Ecclesiæ Catholicæ Canadensis Inc., 1973
Ce document est protégé par la loi sur le droit d'auteur. L'utilisation des services d'Érudit (y compris la reproduction) est assujettie à sa politique d'utilisation que vous pouvez consulter en ligne.

https://apropos.erudit.org/fr/usagers/politique-dutilisation/ 


\section{L'Église canadienne à partir de la formation de la province ecclésiastique de Québec (1844) jusqu'au cinquième Concile provincial de Québec (1873). Evolution des structures et mentalité des évêques.}

Le titre que nous avons donné à notre causerie est un peu élaboré; il demande quelques explications. Nous partons de 1844, date de la formation de la province ecclésiastique de Québec, c'est-à-dire du moment où Rome a accepté de regrouper les évêques du Canada-Uni autour d'un archevêque à Québec. Et nous nous rendons en 1873, alors que la province ecclésiastique de Québec sera réduite pratiquement aux limites de la province civile de Québec.

Durant ces trente années, l'Église canadienne s'est complètement transformée. Nous retenons deux aspects seulement de cette évolution: la création de nouveaux diocèses ou de nouvelles circonscriptions ecclésiastiques et deuxièmement l'attitude des évêques de l'époque, face à l'autorité civile, face aux tensions entre francophones et anglophones et enfin, aux prises avec de graves divergences de mentalité au sein même de l'épiscopat.

\section{Rappel: de la conquête à 1844.}

Il faut cependant rappeler brièvement, en remontant encore plus loin dans le passé, qu'avec la conquête anglaise, l'existence même de l’Église catholique au Canada avait été mise en péril. $\mathbf{M}^{\mathrm{gr}}$ de Pontbriand, évêque de Québec, et seul évêque des colonies françaises en Amérique du Nord, était mort le 8 juin 1760, deux mois avant la capitulation de Montréal. Le conquérant, malgré les promesses et les traités, n'était guère disposé à laisser nommer un nouvel évêque catholique à Québec et à favoriser le développement d'une religion qui était particulièrement honnie et hors la loi en Angleterre.

L'abbé Lucien Lemieux a raconté dans son ouvrage sur L'établissement de la première province ecclésiastique au Canada, 1783-1844 (Montréal, 1968), comment, à force de patience, de chance et de courage, l’Église catholique a réussi à se maintenir dans un "Canada " devenu anglais. Vous en connaissez comme moi les grandes étapes. On obtient d'abord un évêque à Québec en la personne de $\mathrm{M}^{\mathrm{gr}}$ Briand, mais 
sans titre officiel. Puis, cet évêque se fait donner un coadjuteur avec droit de succession et avec entente que les futurs évêques do Québec pourront également se faire nommer un coadjuteur. La succession permanente d'un évêque à Québec est ainsi assurée.

Mais il faut bientôt songer à diviser le diocèse de Québec. L'étendue du territoire est déjà excessive et la population augmente rapidement. Après bien des essais infructueux, on obtient (1818-1820) des districts où résideront des évêques auxiliaires de celui de Québec. La Nouvelle-Écosse cependant devient tout de suite un vicariat séparé (1817). Puis, les divers districts accèdent un à un au statut de diocèses indépendants. Ce qui fait penser à la nécessité de regrouper ces territoires d'une nouvelle façon, c'est-à-dire par la formation d'une province ecclésiastique.

Pour chacune de ces étapes, on n'avait rien entrepris sans avoir auparavant obtenu l'assentiment des autorités civiles locales et londoniennes, sans parler de celle de Rome. $\mathrm{M}^{\mathrm{gr}}$ Lartigue, premier évêque de Montréal, qui avait étudié le droit et qui possédait un tempérament de chef, se serait bien passé de certaines formalités et aurait, s'il avait pu décider sans passer par l'évêque de Québec, placé l'autorité civile devant le fait accompli. C'est ainsi qu'il écrivait à l'abbé Tabeau, le 4 novembre 1829: "Si j'avais maintenant une bulle de Rome qui érigea Montréal en évêché et m'en nommât évêque, je me moquerais du reste ${ }^{1}$. " $M^{\mathrm{gr}}$ Bourget, son successeur, héritera de cette même détermination. Il faut dire que l'esprit de l'époque tendait à la séparation de l'Église et de l'État ou du moins à une certaine indépendance des Églises par rapport à la puissance civile.

La création du diocèse de Toronto en 1841 se réglera à Rome, avant que Londres ou le Gouverneur ait donné leur assentiment ${ }^{2}$. Le nouvel élu, l'abbé Michael Power, qui devenait ainsi évêque de Toronto, était auparavant curé de Laprairie mais il avait passé une bonne partie de sa vie en Nouvelle-Écosse. Il était bien vu du gouverneur Sydenham ${ }^{3}$. Depuis $\mathrm{M}^{\mathrm{gr}}$ Plessis, lorsqu'un nouvel évêque était nommé, il devait prêter serment de fidélité à sa Majesté britannique entre les mains du gouverneur. $\mathrm{M}^{\mathrm{gr}}$ Power, enhardi peut-être par ses bonnes relations avec l'autorité civile, et se situant sans doute dans la mentalité d'indépendance de ses collègues de Montréal, retarda de remplir cette formalité ou ce devoir. Il en fit même un objet de marchandage, disant qu'il prêterait le serment lorsqu'on lui aurait accordé une corporation épiscopale. Or seul le diocèse de Montréal avait obtenu cette reconnaissance

1 ACAM, RLL 5, 4 nov. 1829.

2 LEMIEUX, Etablissement, 472-473.

3 Ibid. 
civile en 1839, avec le droit de posséder en mainmorte. Même Québec ne l'avait pas encore. C'est en 1845 seulement que Toronto l'obtiendra en même temps que Québec et Kingston ${ }^{4}$. $M^{\text {gr }}$ Power mourra du typhus deux ans plus tard, en 1847, sans jamais avoir prêté le serment d'allégeance ${ }^{5}$.

C'est aussi pour renforcer cette indépendance par rapport à l'autorité civile que $\mathrm{M}^{\mathrm{gr}}$ Lartigue, et d'autres déjà avant lui, avaient eu l'idée de créer une province ecclésiastique. Déjà les évêques américains se réunissaient en conciles à Baltimore depuis 1829. Ici, l'on songeait même à inclure les provinces maritimes dans la province ecclésiastique de Québec. On avait sondé à plusieurs reprises l'opinion des évêques de l'Atlantique. On avait presque obtenu leur consentement. Cependant, lorsque la province ecclésiastique de Québec sera enfin créée en 1844, elle ne regroupera que quatre diocèses: Québec, Montréal, Kingston et Toronto, c'est-à-dire tous les diocèses du Canada-Uni. L'évêque de Halifax, $M^{\mathrm{gr}}$ William Walsh (1842), avait fait échec à un plus large rassemblement. Mais $\mathrm{M}^{\mathrm{gr}}$ Bourget, qui avait succédé en 1840 à $\mathbf{M}^{\mathrm{gr}}$ Lartigue à Montréal et qui était devenu l'artisan principal de la formation de cette province ecclésiastique, ne désespérait pas d'arriver à rattacher les diocèses des provinces maritimes ainsi que les missions du Nord-Ouest à l'archidiocèse de Québec.

$\mathrm{M}^{\mathrm{gr}}$ Lartigue avait toujours regretté pour sa part la nomination d'évêques étrangers, comme celle de l'Écossais Alexander Macdonnell à Kingston (1818), à la tête des nouveaux diocèses découpés sur celui de Québec. D'autres Écossais ou Irlandais avaient été nommés dans les provinces maritimes. $\mathbf{M}^{\mathrm{gr}}$ Lartigue craignait que l'ancienne discipline ecclésiastique du diocèse de Québec soit toute modifiée et qu'il devienne de plus en plus difficile de regrouper tous les diocèses de l'Amérique britannique du Nord en une seule province ecclésiastique. C'est pourquoi il avait sauté sur l'occasion lorsque l'évêque de Kingston, $\mathbf{M}^{\mathrm{gr}}$ Macdonnell, désespérant de se trouver un coadjuteur en Europe, avait demandé aux évêques du Bas-Canada s'ils ne pourraient pas lui en fournir un. L'abbé Rémi Gaulin était ainsi devenu en 1833 évêque coadjuteur et bientôt évêque titulaire de Kingston, alors seul diocèse catholique du HautCanada ${ }^{6}$.

4 Ibid., 527, note 35 .

5 C'est du moins ce qu'affirme Monsieur Quiblier, p.s.s., dans une lettre à son Supérieur Général à Paris le 13 septembre 1850, Archives Saint-Sulpice à Paris, 100-I, 166.

6 Le nouvel élu parlait couramment l'anglais. Le gouvernement et le clergé du Haut-Canada n'avaient pas vu d'un très bon oeil cette nomination. La création du diocèse de Montréal, trois ans plus tard, avec $\mathbf{M}^{\mathrm{gr}}$ Lartigue comme évêque, inquiétera aussi le gouvernement. Cela donnait trop de puissance à une Eglise qu'on aurait souhaité à la longue voir disparaître. 
Par ailleurs, $\mathrm{M}^{\mathrm{gr}}$ Macdonald, évêque de Charlottetown depuis 1837, s'était montré sympathique à l'idée d'appartenir à la province ecclésiastique de Québec. Mais il y mettait une condition: il ne le demanderait à Rome que si les autres évêques des " maritimes " le désiraient également. Or, l'évêque de Halifax, $M^{g r}$ Walsh, réclamait plutôt maintenant une province ecclésiastique indépendante pour les diocèses des " maritimes". On pouvait presque désespérer de jamais réunir ces territoires à l'archidocèse de Québec.

\section{De 1844 à 1851}

$\mathrm{M}^{\mathrm{gr}}$ Bourget ne se décourage pas pour autant. Dans son deuxième voyage à Rome (1846-1847), il obtient la création du diocèse de Bytown (Ottawa) qui est rattaché à l'archidiocèse de Québec; puis, il fait déclarer Saint-Jean de Terre-Neuve membre de la province ecclésiastique de Québec, sans doute parce que son évêque, $M^{\text {gr }}$ Fleming, avait montré une certaine ouverture à cette idée ${ }^{7}$. En même temps, le vicariat apostolique de la Baie James et de la Baie d'Hudson, créée en 1844, devenait le vicariat du Nord-Ouest (1847), toujours sous la gouverne de $\mathrm{M}^{\mathrm{gr}}$ Provencher. Il était lui aussi rattaché à l'archidiocèse de Québec. La province ecclésiastique de Québec comptait donc désormais six diocèses et un vicariat: Québec, Montréal, Bytown, Kingston, Toronto et Terre-Neuve, plus le vicariat du Nord-Ouest.

C'est $\mathrm{M}^{\mathrm{gr}}$ Bourget qui menait ainsi la marche tambour battant, mais $\mathrm{M}^{\mathrm{gr}}$ Signay, l'archevêque de Québec, ne le suivait que de très loin. Déjà, l'évêque de Montréal avait dû arracher le consentement de son collègue de Québec pour obtenir la création de la province ecclésiastique en 1844. Depuis, $\mathbf{M}^{\mathrm{gr}}$ Signay ne se décidait pas à porter le titre d'archevêque de peur de mécontenter le gouvernement. Il ne se pressait pas non plus de réunir en concile les évêques qui dépendaient désormais de lui, comme les lois de l’Église le lui prescrivaient.

Découragé de rien obtenir de ce côté, $M^{\mathrm{rg}}$ Bourget avait décidé de dénoncer à Rome l'inertie de l'archevêque et d'obtenir si possible son remplacement. C'est ce qu'il fit en 1847 en remettant de main à main un mémoire au Pape. Pie IX lui aurait répondu: "Vous pouvez dire à l'archevêque de Québec que sa démission plairait au Saint-Père ${ }^{8}$. " $\mathrm{M}^{\mathrm{gr}}$ Signay, offusqué de l'audace de son suffragant de Montréal, attendra que Rome lui signifie par écrit sa volonté. Mais $\mathrm{M}^{\mathrm{gr}}$ Bourget revenant à la charge, il finira par envoyer au Saint-Siège une lettre de démission ${ }^{9}$.

Il remit alors à son coadjuteur, $\mathrm{M}^{\mathrm{gr}}$ Turgeon, l'administration de l'archidiocèse. Normalement ce dernier recevait ainsi tous les pouvoirs

7 Lemieux, Etablissement, 522.

8 Bourget à Signay, 25 fév. 1847, AAQ, DM, 9: 42.

9 Bourget à Guigues, 27 sept. 1849, ACAM, RLB 5: 303. 
de l'archevêque, mais d'accord avec $\mathbf{M}^{\mathrm{gr}}$ Bourget, il voulut faire préciser par Rome s'il avait aussi le pouvoir de convoquer un concile provincial. En attendant une réponse, $\mathrm{M}^{\mathrm{gr}}$ Bourget et $\mathrm{M}^{\mathrm{gr}}$ Turgeon s'entendent pour organiser une simple réunion d'évêques en vue de régler quelques problèmes urgents et de préparer les assises conciliaires ${ }^{10}$.

La réunion s'ouvrit le $1^{\text {er }}$ mai 1850 à Montréal. Elle groupait les évêques Turgeon, Bourget, Phelan, administrateur de Kingston tenant la place du titulaire Rémi Gaulin qui souffrait d'une sorte de dépression nerveuse, Prince, coadjuteur de Montréal et Guigues, évêque de Bytown. Le siège de Toronto était vacant depuis 1847. Les évêques du NordOuest et de Terre-Neuve avaient été jugés trop éloignés pour être convoqués.

On n'avait demandé aucune permission au gouvernement pour se rencontrer. On conseillera cependant à $\mathbf{M}^{\mathrm{gr}}$ de Charbonnel, dont on apprendra l'élection au siège de Toronto durant cette réunion, d'écrire au Gouverneur avant d'aller se présenter à lui en personne lors de son retour au Canada. On se chargeait entre-temps d'annoncer à Lord Elgin la décision romaine ${ }^{11}$. On protestait toujours alors, selon une formule devenue un peu rituelle, de la loyauté du nouvel élu envers la couronne britannique. En 1847, le gouvernement avait formulé quelques remarques lorsqu'il avait appris la nomination de $\mathrm{M}^{\mathrm{gr}}$ Guigues comme premier évêque de Bytown. Pouvait-on se fier, demandait le gouverneur, à l'allégeance britannique d'un Français qui n'était au pays que depuis quelques années. $\mathrm{M}^{\mathrm{gr}}$ Bourget avait dû protester alors à nouveau de la loyauté du nouvel élu et de son attachement pour son pays d'adoption ${ }^{12}$.

Tel que prévu, les évêques réunis à Montréal prirent quelques décisions communes, comme de défendre la lecture des bibles et «tracts» distribués par les protestants, d'approuver la fondation du True Witness, journal catholique de langue anglaise qui sera publié à Montréal; d'encourager la fondation de bibliothèques paroissiales, etc. Ils se sont aussi nommés un délégué permanent à Rome en la personne de Charles-François Baillargeon, curé de Québec. Ils déterminèrent surtout une liste de questions à traiter dans le prochain concile: révision des livres liturgiques et du catéchisme, développement de l'éducation chrétienne, formation des clercs, réforme des mours, etc. Puis ils fixèrent la date d'ouverture de cette assemblée au 15 août $1851^{13}$.

10 Bourget aux évêques, 9 avril 1850, ACAM, RLB 6: 57.

11 Procès-verbal de la réunion, ACAM, 272.101.

12 Bourget à Elgin, 24 juillet 1848, ACAM, RLB 4: 529.

13 Procès-verbal de la réunion, ACAM, 272.101. 


\section{3. $I^{\text {er }}$ concile, 1851}

$\mathrm{M}^{\text {gr }}$ Signay, archevêque de Québec, mourait quelques mois plus tard, le 3 octobre 1850. $\mathbf{M}^{\mathrm{gr}}$ Turgeon n'avait plus désormais à se demander s'il avait le pouvoir de convoquer le concile. Le 6 janvier 1851, il publiait l'édit officiel de convocation du premier concile provincial de Québec. On n'en avait même pas averti le gouvernement. Dans son mandement, $M^{\mathrm{gr}}$ Turgeon célébrait la liberté nouvelle dont jouissait l'Église catholique dans le monde ${ }^{14}$.

Les évêques des "maritimes", sans faire partie de la province ecclésiastique de Québec, sauf Terre-Neuve, devaient, d'après une décision romaine, se rendre aux conciles provinciaux de Québec. En envoyant la convocation au nouvel évêque de Terre-Neuve, $\mathbf{M}^{\mathrm{gr}}$ Thomas Mullock, $\mathbf{M}^{\mathrm{gr}}$ Turgeon prenait sur lui d'ajouter que si, comme son prédécesseur, il désirait se séparer de la province ecclésiastique de Québec, l'archevêque n'y mettrait pas d'obstacle ${ }^{15}$. $M^{\mathrm{gr}}$ Turgeon craignait, comme d'ailleurs son prédécesseur $\mathrm{M}^{\mathrm{gr}}$ Signay, la présence d'un trop grand nombre d'évêques anglophones aux futurs conciles de Québec.

$\mathrm{M}^{\mathrm{gr}}$ Turgeon prévoyait bien que les évêques de Bytown, de Kingston et de Toronto ne pourraient plus longtemps être francophones. Il était même invraisemblable que les titulaires des trois diocèses du HautCanada aient pu être un moment tous trois francophones ${ }^{16}$. Les évêques cependant n'avaient pas fait exprès pour faire nommer ces trois prélats de langue française dans les diocèses du Haut-Canada. Le premier évêque de Toronto, $\mathrm{M}^{\mathrm{gr}}$ Power, était anglophone. Et pour le remplacer, on avait suggéré en premier lieu le nom du Père Larkin, jésuite vivant aux Etats-Unis mais ancien sulpicien de Montréal. Toutes les pressions cependant pour faire accepter l'épiscopat à ce dernier avaient abouti à un échec. C'est donc le second candidat en liste, M. de Charbonnel, en réalité le candidat de $\mathrm{M}^{\mathrm{gr}}$ Bourget, qui avait été nommé. Au diocèse de Kingston, c'est $M^{\mathrm{gr}}$ Macdonnell qui avait accepté un canadien-français pour lui succéder. Quant au diocèse de Bytown, c'est surtout parce qu'il desservait les missions indiennes de l'Outaouais et de la Baie James qu'on y avait nommé un Oblat, dont la congrégation, espérait-on, pourrait fournir plus facilement les missionnaires nécessaires pour cet

14 MEQ, 3: 19-20.

15 Turgeon à Mulloch, 27 janvier 1851, AAQ, RL 23: 612.

16 Turgeon à Bourget, 25 février 1851, AAQ, RL 23: 649. En fait, on peut dire qu'ils ne l'ont jamais été tout-à-fait, car lorsque $\mathrm{M}^{\mathrm{gr}}$ de Charbonnel a été élu évêque de Toronto en 1851, $\mathrm{M}^{\mathrm{gr}}$ Gaulin, évêque de Kingston, avait déjà remis l'administration de son diocèse à son coadjuteur, Mgr Phelan. Mais aussitôt qu'il se sentait mieux, $M^{g r}$ Gaulin cherchait à reprendre en mains les rênes de son diocèse. Ce n'est qu'au premier concile de Québec, en 1851, qu'on le forcera à y renoncer définitivement. 
apostolat. D'ailleurs, près de la moitié des fidèles du diocèse de Bytown étaient de langue française.

Le premier concile provincial de Québec s'ouvrit tel que prévu le 15 août 1851 dans la cathédrale de cette ville. Les dix évêques ainsi rassemblés avaient conscience de poser un geste très important dans l'évolution de l'Église catholique au Canada. Important surtout par l'image nouvelle qu'il allait donner d'une Église catholique puissante et libre dans une colonie anglaise.

Pourtant, des cinq évêques des «maritimes invités au concile, deux (Halifax et Arichat) n'avaient même pas répondu à la lettre de convocation. L'évêque de Halifax s'affairait toujours à obtenir une province ecclésiastique indépendante et se pensait ainsi dispensé de toute obligation de se rendre à Québec. $\mathbf{M}^{\mathbf{b}}{ }^{\mathbf{x}}$ Dollard, évêque de Frédéricton, s’était excusé parce que malade. De fait, il mourra quelque temps après. Seuls les évêques de Saint-Jean, Terre-Neuve, et de Charlottetown se rendirent à Québec. $M^{\mathrm{gr}}$ Mulloch, de Terre-Neuve, apportait un décret romain qui séparait son diocèse de la province ecclésiastique de Québec. Ce qui faisait dire à son compagnon de Charlottetown qu'une province ecclésiastique regroupant les diocèses des " maritimes " était déjà acceptée en principe par le Saint-Siège ${ }^{17}$. Ces deux évêques, ne faisant pas partie de la province ecclésiastique de Québec, n'avaient que voix consultative dans les réunions du concile. Dès le deuxième jour des assises, ils commencent à s'absenter et au lendemain de la deuxième session publique, à laquelle $\mathrm{M}^{\mathrm{gr}}$ Mulloch devait prêcher en anglais, ils retourneront tous deux dans leur diocèse respectif ${ }^{18}$. Le concile allait poursuivre ses assises sans eux pendant une autre semaine.

Le rêve de $\mathrm{M}^{\mathrm{gr}}$ Lartigue et l'obstination de $\mathrm{M}^{\mathrm{gr}}$ Bourget de réunir tous les évêques de l'Amérique britannique du Nord, étaient à jamais envolés. On peut sérieusement se demander d'ailleurs si $M^{\mathrm{gr}}$ Lartigue n'aurait pas modifié sa position avec le temps et ne se serait pas rallié un peu à l'opinion de $\mathbf{M}^{\mathrm{gr}}$ Signay et de $\mathbf{M}^{\mathrm{gr}}$ Turgeon devant l'évolution de la situation. Il n'avait certes pas prévu que le nombre des évêques anglophones pourrait dépasser assez rapidement celui des évêques de langue française dans les futurs conciles.

Une des seules décisions du premier concile qui va modifier les structures de l'Église canadienne est l'accord des évêques pour demander à Rome la création prochaine de deux nouveaux diocèses de langue

17 De fait, la province ecclésiastique de Halifax sera créée dès l'année suivante, en 1852, sans inclure cependant Terre-Neuve qui préférera demeurer rattachée directement au Saint-Siège, APFR, Acta (1852) 61.

18 Acta et decreta primi concilii... (Québec, 1852), 27 et 32. 
française, l'un à Saint-Hyacinthe, l'autre aux Trois-Rivières. L'érection de ces deux diocèses, obtenue dès 1852 , va modifier pour un temps l'équilibre de la province ecclésiastique de Québec ${ }^{19}$. Celle-ci compte maintenant sept diocèses et un vicariat: Québec, Montréal, SaintHyacinthe et Trois-Rivières dans le Bas-Canada; Kingston et Toronto dans le Haut-Canada; Bytown entre les deux, et le vicariat du NordOuest. Seul l'administrateur de Kingston, $\mathrm{M}^{\mathrm{gr}}$ Patrick Phelan, est anglophone ${ }^{20}$.

$\mathrm{M}^{\mathrm{gr}}$ de Charbonnel, l'évêque de Toronto, réussit, dès 1853 , à se faire nommer un coadjuteur en la personne de M. Patrick Dowd, son ancien confrère au Séminaire Saint-Sulpice de Montréal ${ }^{21}$. Mais à la nouvelle de sa nomination, l'élu avait disparu de la circulation. Pas moyen de le retrouver. L'évêque de Toronto, même s'il soupçonnait le supérieur du Séminaire de Montréal, Monsieur Bilaudèle, d'être un peu complice dans l'affaire, dut se contenter d'alerter les autorités supérieures. Il écrivit en Irlande, à Paris et à Rome, afin qu'un ordre fût donné à ce prêtre d'accepter sa nomination. Un mois plus tard, $M$. Dowd réapparaissait à Montréal, mais ni $\mathbf{M}^{\mathrm{gr}}$ Charbonnel, ni $\mathbf{M}^{\mathrm{gr}}$ Bourget, ni même une lettre de Rome ne réussirent à persuader l'intéressé d'accepter l'épiscopat. L'évêque de Toronto dut se résigner à poursuivre sa tâche seul pour quelque temps, alors qu'il songeait déjà à remettre l'administration de son diocèse au nouveau coadjuteur ${ }^{22}$.

$\mathrm{M}^{\mathrm{gr}}$ de Charbonnel se faisait presque un complexe de ne pas parler très bien l'anglais alors que Toronto remplaçait de plus en plus Kingston comme centre du Canada anglais. De plus, il avait dû sévir contre plusieurs prêtres irlandais de son diocèse dont la conduite était loin d'être édifiante à ses yeux. Il en avait même expulsé une douzaine. Il est vrai qu'il réussira par la suite à en recruter en Europe, surtout en France, trois fois plus. Mais il s'était fait des ennemis chez certains

19 On ne mettra encore une fois le gouvernement au courant que lorsque les deux diocèses auront été érigés par Rome. Les titulaires en étaient $\mathbf{M}^{\mathrm{gr}}$ Prince pour Sainte-Hyacinthe, lequel était auparavant coadjuteur à Montréal. Cela entrainait la nomination d'un nouveau coadjuteur pour Montréal et c'est le chanoine Joseph LaRocque qui fut choisi. Le nouvel évêque des Trois-Rivières était l'abbé Thomas Cooke, curé de cette même ville depuis 17 ans.

20 Il était aussi bilingue, ayant fait partie longtemps du Séminaire de Saint-Sulpice de Montréal. Il avait été nommé, d'après Monsieur Quiblier, son supérieur d'alors, par l'influence de $\mathrm{M}^{\mathrm{gr}}$ Bourget et restait un instrument docile entre les mains de ce dernier. Il faut sans doute nuancer ce jugement de $\mathbf{M}$. Quiblier écrit en 1851, cinq ans après avoir été forçé de démissionner comme supérieur à Montréal par l'intervention de $\mathbf{M}^{\mathrm{gr}}$ Bourget, Quiblier à Barnabo, 8 décembre 1851, APFR, SC (1849-1857) 390.

21 LaRocque à Bourget, 14 mars 1853, ACAM, 901.077.

22 Charbonnel à Guigues, 16 avril 1853, AAO, RL 5: 193. M. Dowd conservera son poste de curé ou desservant des Irlandais de Montréal durant de très nombreuses années. 
autres. Il s'était aussi brouillé avec le gouvernement, dans ses relations en particulier avec le surintendant de l'éducation Ryerson, en luttant pour avoir des écoles catholiques "séparées". Enfin, il avait des aspirations mystiques et voulait se faire moine. Son clergé et les fidèles de son diocèse trouvaient que sa tenue vestimentaire et le mobilier de son évêché, réduits au minimum par esprit de pauvreté, manquaient quelque peu de decorum face à des évêchés ou des églises protestantes prospères.

$\mathrm{M}^{\mathrm{gr}}$ de Charbonnel était apparenté à la haute noblesse française. Il avait liquidé à même son argent personnel une bonne partie de la dette dont était grevée la cathédrale de Toronto construite par son prédécesseur. Il avait un caractère entier qui le faisait s'attacher avec acharnement à la défense de certains principes. Il avait aussi parfois des sautes d'humeur surprenantes. On raconte qu'un jour, en pleine cérémonie dans sa cathédrale, il avait jeté sa mitre par terre parce que son cérémoniaire tardait trop à la lui enlever ${ }^{23}$. Ses prêtres reconnaissaient toutefois que ce tempérament de feu avait été utile pour rétablir la discipline ecclésiastique dans un diocèse demeuré sans évêque pendant trois ans. On admettait aussi que nul autre que lui aurait pu lutter avec autant d'acharnement pour obtenir des écoles catholiques.

Entre temps, dans les missions du Nord-Ouest, $\mathbf{M}^{\text {gr }}$ Norbert Provencher, évêque de Saint-Boniface, expirait ( 7 juin 1853) assisté par l'abbé Laflèche, le futur évêque des Trois-Rivières. $\mathrm{M}^{\mathrm{gr}}$ Taché, le coadjuteur, se trouvait alors à près de 500 milles au nord-ouest de la Rivière-Rouge ${ }^{24}$. Il devenait évêque de Saint-Boniface à l'âge de 29 ans et le restera pendant plus de 40 ans. Comme tous les Oblats de l'époque, il voyait en $\mathrm{M}^{\mathrm{gr}}$ Bourget un père, un protecteur et comme le fondateur de sa communauté au Canada. Il lui restera toujours très attaché. Il demeurera intimement lié aussi à $M^{\text {gr }}$ Laflèche avec qui il avait partagé les durs labeurs de la vie missionnaire.

\section{Deuxième concile, 1854}

D'après le concile de Trente, les conciles provinciaux devaient se tenir tous les trois ans. Un second concile de Québec était en effet prévu pour mai 1854. Les évêques cependant ne manifestent déjà plus le même enthousiasme qu'en 1851 à l'approche de cette nouvelle réunion. $\mathrm{M}^{\mathrm{gr}}$ Turgeon, l'archevêque de Québec, suggère à son collègue de Montréal que la rencontre ne dure qu'une semaine au lieu de deux, et que chaque évêque ne soit accompagné que de deux théologiens alors qu'ils en

23 Philbert à Barnabo, mars 1860, mémoire sur le diocèse de Toronto. Il semble bien que le caractère de l'évêque de Toronto avait été l'une des raisons qui avaient amené $M$. Dowd à refuser l'épiscopat.

24 Taché à Turgeon, 23 décembre 1853, AAQ, Conc. pr. 2. 
avaient trois au premier concile. L'archevêque avouait en plus qu'il n'avait pas beaucoup de suggestions à faire sur les problèmes que l'on pourrait débattre à cette assemblée ${ }^{25}$.

C'est l'évêque de Montréal qui vient à la rescousse. Il suggère une foule de questions que $M^{g r}$ Turgeon croit devoir séparer tout de suite en deux catégories: celles qui pourraient faire l'objet de décrets, et les autres sur lesquelles on pourrait émettre tout au plus des règlements ${ }^{26}$.

Pour ce qui nous intéresse ici en particulier, c'est-à-dire l'évolution des structures de l'Église canadienne et la mentalité de ses pasteurs, retenons que $\mathrm{M}^{\mathrm{gr}}$ de Charbonnel ne demande plus seulement un coadjuteur mais la division de son diocèse en trois parties, c'est-à-dire la création de deux nouveaux sièges épiscopaux, dont l'un serait à Hamilton et l'autre à London, tous deux à l'ouest de Toronto ${ }^{27}$. La population de ces deux villes, au dire de l'évêque, avait doublé en moins de cinq ans.

$M^{\text {gr }}$ Taché, pour sa part, bien que ne pouvant se rendre à Québec, - les voyages entre la Rivière-Rouge et le "Canada étant à peu près impraticables au printemps - demandait à ses collègues de préciser, si possible, le tracé de la frontière est de son diocèse. Il se proposait de demander ensuite à Rome la création d'un vicariat apostolique dans cette région ${ }^{28}$. Ce vicariat renfermerait les territoires à l'ouest de la Baie James et de la Baie d'Hudson et pourrait englober la partie nord des diocèses de Toronto et de Bytown ${ }^{20}$.

Lorsque les diocèses de Hamilton et de London auront été établis par le Saint-Siège en 1856, le Haut-Canada groupera quatre diocèses, le même nombre que dans le Bas-Canada, Ottawa demeurant toujours entre les deux. Le nouvel évêque de Hamilton était un Irlandais, $\mathbf{M}^{\mathbf{g r}^{\mathrm{r}}}$ John Farrell, celui de London, un Canadien-français, $\mathbf{M}^{\mathrm{gr}}$ Adolphe Pinsonnault. On peut se demander comment il se fait qu'on ait nommé encore un évêque francophone dans un diocèse de cette région. Il faut dire d'abord que les évêques, lors du deuxième concile, s'étaient refusés à présenter de nouveau à Rome le nom de Patrick Dowd, même s'il s'agissait de le faire nommer cette fois dans un autre diocèse que Toronto.

25 Turgeon à Bourget, 12 décembre 1853, AAQ, RL 25: 320.

26 AAQ, Conseil particulier de l'archevêque: procès-verbaux des réunions, $1^{\text {er }}$ mars 1854 .

27 Charbonnel à Turgeon, 20 février 1854, AAQ, 320CN, 7: 34.

28 Charbonnel à Franzoni, APFR, SOCG (1856) 87.

29 Taché à Turgeon, 23 décembre 1853, AAQ, Conciles provinciaux II. Le vicariat projeté sera créé en 1862 et portera le nom d'Athabaska-Mackenzie. Mgr Henri Faraud, o.m.i., en sera le titulaire. L'évêque de Saint-Boniface avait songé d'abord à confier ce vicariat aux Jésuites qui avaient des missions au nord du lac Supérieur, Taché à Turgeon, 23 décembre 1853, AAQ, Conciles provinciaux II. 
On s'était assuré par ailleurs du consentement du chanoine Pinsonnault qui était présent au concile. Il faut dire aussi que l'évêque de Toronto affirmait qu'il n'y avait aucun prêtre anglophone de son diocèse, ni même de tout le Haut-Canada, qui pouvait être présenté pour l'épiscopat. Se cherchant toujours un coadjuteur, il songeait alors à un prêtre de la Nouvelle-Écosse dont il avait entendu vanter les qualités. Il finira par choisir un religieux d'origine irlandaise, supérieur d'un collège à Niagara aux Etats-Unis, le Père John J. Lynch ${ }^{30}$.

$M^{g r}$ Pinsonnault, qui devenait évêque de London, était encore un candidat de $\mathrm{M}^{\mathrm{gr}}$ Bourget et un ancien confrère de $\mathrm{M}^{\mathrm{gr}}$ de Charbonnel. On pourrait dire que, de même que l'évêque de Montréal ne craignait pas la présence d'une majorité d'évêques anglophones dans les conciles de Québec, de même il semble ici avoir fait peu de cas des réactions que pouvait susciter la nomination d'un évêque canadien-français dans le Haut-Canada. Mais les prêtres de cette région, aussitôt qu'ils apprirent qu'un évêque francophone allait être nommé à London, organisèrent un mouvement de protestation ${ }^{31}$.

L'abbé James Chisholm, théologien aux deux conciles de Québec et ancien élève du collège de la Propagande à Rome, se fait le porteparole de ses confrères auprès du cardinal Franzoni ${ }^{32}$. Le Saint-Siège demande alors des explications aux évêques canadiens mais considère cette opposition du clergé du Haut-Canada comme une manifestation d'esprit national ou racial qui ne devrait pas exister. La Propagande va même jusqu'à conseiller aux évêques de la province ecclésiastique de Québec d'avoir un Grand Séminaire unique où les futurs prêtres anglophones et francophones seraient formés ensemble, de façon à se mieux connaître et à mieux s'accorder par la suite ${ }^{33}$. Les évêques répondront en proposant que ce soit le Grand Séminaire de Montréal, qui venait d'être reconstruit au flanc de la montagne, qui rende ce service, sans cependant supprimer les autres Grands Séminaires déjà existants ${ }^{34}$.

30 Charbonnel à Franzoni, 10 juin 1854, APFR, SOCG (1855) 1217. L'évêque de Toronto songeait toujours à démissionner aussitôt qu'il aurait un coadjuteur anglophone. Il avait même pensé à la formation d'une province ecclésiastique indépendante pour le Haut-Canada, Franzoni à Charbonnel, 22 janvier 1853, AAQ, $320 \mathrm{CN} 7: 30$.

31 Il semble bien que le chanoine Pinsonnault se préparait ostensiblement à l'épiscopat dès avant sa nomination, Chisholm à Franzoni, $1^{\text {er }}$ octtobre 1854, APFR, SC (1849-1857) 661.

32 Ibid., le curé de London, l'abbé Gordon, refusera pour sa part, de céder son église à Mgr Pinsonnault pour qu'elle devienne la cathédrale, tel que les évêques l'avaient prévu lors du deuxième concile. Il faudra que ceux-ci menacent le curé de lui enlever tous ses pouvoirs de prêtre pour qu'il accepte de quitter London. Procès-verbal d'une réunion des évêques à Montréal, 19 mai 1856, $26 \mathrm{CP}$ 10: 172 .

33 Barnabo à Baillargeon, 27 mars 1856, APFR, Indice Lettere (1856) Am. Sett. Prov. del Can., 159.

34 Baillargeon à Barnabo, 6 juin 1856, AAQ, RL 26: 230. 


\section{Troisième concile, 1863}

Le 19 février 1855, l'archevêque de Québec, $\mathrm{M}^{\mathrm{gr}}$ Pierre-Flavien Turgeon, était frappé de paralysie alors qu'il célébrait des funérailles dans l'église Saint-Jean-Baptiste de Québec. C'est son coadjuteur, $\mathbf{M}^{\text {gr }}$ Charles-Francois Baillargeon, qui prend alors la relève en assumant l'administration de l'archidiocèse. Il devenait aussi responsable de toute la province ecclésiastique ${ }^{35}$.

$\mathrm{M}^{\mathrm{gr}}$ Baillargeon était un homme modéré, comme l'avait été d'ailleurs $\mathrm{M}^{\mathrm{gr}}$ Turgeon. Cependant, l'archevêque paralysé avait collaboré plus facilement avec $\mathrm{M}^{\mathrm{gr}}$ Bourget, ou s'était montré plus conciliant avec ce dernier, que ne le sera $\mathrm{M}^{\mathrm{gr}}$ Baillargeon. Le nouvel administrateur de Québec avait une santé délicate et n'était pas ce que l'on peut appeler un chef naturel. Mais il ne pouvait supporter les ultramontains intransigeants. Par ailleurs, la charge de l'archidiocèse lui paraîtra lourde; il offrira plusieurs fois sa démission. Il essaiera d'éviter d'assumer la responsabilité de toute la province ecclésiastique, surtout celle de la convocation des conciles.

Normalement il aurait dû y avoir un troisième concile en 1857 , trois ans après le deuxième. $\mathbf{M}^{\mathrm{gr}}$ Baillargeon pouvait prétexter qu'il n'avait pas le pouvoir de le convoquer ou qu'il ne voulait pas le demander, par respect pour l'archevêque malade. Il craignait surtout l'influence envahissante et l'ultramontanisme militant de l'évêque de Montréal.

Trois ans plus tard, en $1860, \mathrm{M}^{\mathrm{gr}}$ Baillargeon est bien obligé de se poser à nouveau le problème. Il demande alors timidement au Saint-Siège si, dans sa situation, il est tenu de convoquer un concile. On lui répond d'en juger selon les décrets de Benoit XV sur les synodes diocésains ${ }^{36}$. Ce renvoi semble avoir dégagé l'administrateur de toute responsabilité puisqu'il ne parle plus de convoquer le concile pour 1860 . Il faudra attendre un autre triennat.

Mais entre temps, certaines critiques circulent parmi le clergé du fait que $\mathrm{M}^{\mathrm{gr}}$ Baillargeon ne se considère que comme un administrateur du diocèse de Québec et ne s'occupe aucunement des besoins de la province ecclésiastique. D'autant plus que certains problèmes étaient devenus aigus et auraient demandé une intervention ${ }^{37}$. D'Arcy McGee, par exemple, journaliste irlandais émigré aux États-Unis, puis installé récemment à Toronto, venait de lancer un nouveau journal catholique, The Canadian Freeman, avec l'approbation de $\mathrm{M}^{\mathrm{gr}}$ de Charbonnel.

35 MEQ 4: 9. $\mathrm{M}^{\mathrm{gr}}$ Baillargeon avait alors 56 ans.

36 Dubbi proposés par $\mathrm{M}^{\mathrm{gr}}$ Baillargeon, $1^{\text {er }}$ décembre 1860, APFR, SC (1858-1861) 492 .

37 Philbert à Barnabo, 18 avril 1861, APFR, SC (1858-1861) 615. 
Or, $\mathrm{M}^{\mathrm{gr}}$ Bourget avait tout de suite vu dans ce journal un rival du True Witness, lequel était censé être le journal catholique officiel de langue anglaise pour toute la province ecclésiastique ${ }^{38}$. Ce qui envenimait les choses, c'est que D'Arcy McGee s'était fait élire comme député réformiste, et donc libéral, et qu'il esseyait de réconcilier l'Église avec les libéraux et même avec son pire ennemi aux yeux des évêques, un autre journaliste de Toronto, George Brown, propriétaire du Globe ${ }^{39}$.

Une réunion des évêques aurait sûrement éclairci la situation, sinon réglé la question. Mais les deux journaux catholiques, aux tendances si opposées, se faisaient ouvertement la guerre et $\mathrm{M}^{\mathrm{gr}}$ Baillargeon n'intervenait pas.

Un rapport envoyé à Rome en 1861 par un prêtre français, qui travaillait depuis un an pour l'évêque de Toronto, fit que la Propagande proposa à l'administrateur de Québec de lui donner les pouvoirs de convoquer le concile ${ }^{40}$. $\mathrm{M}^{\mathrm{gr}}$ Baillargeon semble n'avoir pas relevé cette suggestion. Mais l'année suivante, en 1862, le Pape invitait à Rome tous les évêques du monde entier, officiellement pour la canonisation de trente martyrs japonais, mais en réalité pour protester plus solennellement à la face du monde contre la situation qui lui était faite en Italie par les partisans de l'unification de la péninsule. Le cardinal Barnabo, devenu préfet de la Congrégation de la Propagande, demande alors à $\mathbf{M}^{\mathrm{gr}}$ Baillargeon pourquoi il refuse de convoquer le concile.

Ce dernier admet que les affaires de la province ecclésiastique sont négligées mais il se dit convaincu qu'il n'y a aucun problème assez urgent pour demander la convocation d'une réunion conciliaire. Les deux premiers conciles, selon lui, ont établi un cadre disciplinaire général qui suffit pour le moment ${ }^{41}$. Plus confidentiellement, $\mathbf{M}^{\mathrm{gr}}$ Baillargeon explique au Préfet qu'il lui semble impossible de s'entendre avec l'évêque de Montréal, principalement sur les questions de liturgie. $\mathbf{M}^{\mathrm{gr}}$ Bourget avait changé certains usages liturgiques dans son diocèse, sous prétexte que cela se faisait ainsi à Rome, même si ça n'était pas prescrit par le cérémonial provincial élaboré par le premier concile de Québec et approuvé ensuite par Rome ${ }^{42}$.

Le cardinal Barnabo décide néanmoins de donner à $\mathrm{M}^{\mathrm{gr}}$ Baillargeon les pouvoirs nécessaires pour convoquer le concile ${ }^{43}$. Les autres

38 Bourget à Charbonnel 30 juin 1858, ACAM, RLB 10: 284.

39 Langevin, secr. à Moylan, rédacteur en chef du Freeman, 28 novembre 1858, ACAM, 255.104, 858-12b; Moylan à Baillargeon, $1^{\text {er }}$ décembre 1858, AAQ 320CN, 6: 88 .

40 Barnabo à Baillargeon, 29 juillet 1861, APFR, Indice Lettere, Am. Sett. Can. in gen., 441 .

41 Baillargeon à Barnabo, 25 mai 1862, AAQ, RL 27: 482.

42 Baillargeon à Barnabo, 5 juin 1862, APFR, SC (1862-1865) 313.

43 AAQ, Conc. pr., Reg. Actes I. 
évêques se diront ensuite d'accord pour que les assises se tiennent en mai 1863. Ce troisième concile de Québec cependant, selon le tempérament de son président, $M^{\mathrm{gr}}$ Baillargeon, évitera d'aborder de front les questions trop controversées, comme celle de la liturgie. $\mathrm{M}^{\mathrm{gr}}$ Bourget, de son coté, lui qui avait été à l'origine de la formation de la province ecclésiastique et de la réunion des conciles de Québec, semble maintenant faire cavalier seul. Il venait par exemple de redemander au Saint-Siège la permission de fonder une université catholique à Montréal, alors que Laval était à peine organisée, mais il n'en parle pas à ses collègues à l'occasion du concile.

L'intransigeance par ailleurs de l'évêque de Montréal compromet de plus en plus son prestige, autant à Rome qu'au Canada. Les nouvelles nominations épiscopales échappent de plus en plus à son influence. Il est étonné, au début de l'année 1864, d'apprendre que le Saint-Siège n'a pas suivi son avis de donner un Oblat comme coadjuteur à $M^{\mathrm{gr}}$ Demers, l'évêque de Vancouver ${ }^{44}$.

Un successeur est donné en 1865 à $\mathrm{M}^{\mathrm{gr}}$ Joseph LaRocque ${ }^{45}$, évêque de St-Hyacinthe pendant que $\mathrm{M}^{\mathrm{gr}}$ Bourget est à Rome. Il ne participe pas aux choix des trois noms qui sont soumis au Saint-Siège. Il sera mécontent de la nomination de l'abbé Charles Larocque au siège de Saint-Hyacinthe. Ce dernier, comme curé de Saint-Jean d'Iberville, avait manifesté une certaine opposition aux réformes liturgiques de son évêque, $\mathrm{M}^{\mathrm{gr}}$ Bourget ${ }^{46}$. Celui-ci avait pourtant fait connaître sur place à Rome son opposition au choix du premier candidat en liste. Il aurait préféré le second, l'abbé Rémi Ouellet, professeur au Séminaire de Saint-Hyacinthe ${ }^{47}$.

L'évêque de Sandwich (auparavant London), $\mathrm{M}^{\mathrm{gr}}$ Adolphe Pinsonnault, est forcé également de démissionner à la fin de l'année $1866^{48}$. Il donne comme raison officielle sa mauvaise santé mais il s'était montré incapable d'administrer son diocèse, et le Saint-Siège avait dû le contraindre à renoncer à son poste ${ }^{49}$. Les évêques essaient alors de nommer

44 On avait nommé plutôt le Père d'Herbomez, o.m.i., vicaire apostolique de la Colombie Britannique, ne laissant à $\mathrm{M}^{\mathrm{gr}}$ Demers que la région immédiate de Vancouver et l'île de Victoria. On avait assigné un séculier, l'abbé Morrisson, du diocèse de Montréal, comme coadjuteur de $\mathbf{M}^{\mathbf{g r}}$ Demers, mais cet abbé refusera l'épiscopat, Bourget à Baillargeon, 21 janvier 1864, ACAM, RLB 13: 362.

45 L'évêque de Saint-Hyacinthe démissionnait pour raison de santé.

46 Truteau à Bourget, 16 avril 1869, ACAM, RLB 18: 266. Voir aussi L..Z. Moreau à Paré, 21 mars 1865, ACAM, 295.103, 865-11 et Ponenza, 29 janvier 1866, APFR, Acta, (1866) 15. Le nom de Charles Larocque avait été mis le premier sur la liste, grâce à l'appui de son cousin démissionnaire $\mathrm{M}^{\mathrm{gr}}$ Joseph LaRocque. A noter par ailleurs que le troisième nom était celui d'Edouard Fabre, qui deviendra plus tard coadjuteur de Mgr Bourget.

47 Ponenza, 29 janvier 1866, APFR, Acta (1866) 15.

48 Baillargeon à Pinsonnault, 14 décembre 1866, AAQ, RL 28: 649.

49 Bourget à Pinsonnault, 17 août 1866, ACAM, RLB 15: 229. 
à sa place un prêtre de langue anglaise, quelqu'un qui travaille déjà dans le diocèse ou au moins dans la région du Haut-Canada. Ils choisissent, sur la suggestion semble-t-il de $\mathbf{M}^{\mathrm{gr}}$ Lynch, l'abbé John Walsh, vicairegénéral de Toronto, lequel sera consacré dans cette dernière ville le 10 novembre $1867^{50}$.

L'abbé Louis-François Laflèche est nommé aussi entre temps coadjuteur de l'évêque des Trois-Rivières (1866). A force d'insister, $\mathbf{M}^{\text {gr }}$ Baillargeon avait réussi à obtenir de $\mathrm{M}^{\mathrm{gr}}$ Cooke, alors âgé de 68 ans et malade depuis plusieurs années, qu'il demande à ses collègues d'envoyer une supplique au Saint-Siège en ce sens. $M^{\mathrm{gr}}$ Bourget n'était sûrement pas opposé au choix de l'abbé Laflèche, mais c'était depuis longtemps le candidat de $\mathbf{M}^{\mathrm{gr}}$ Baillargeon ${ }^{51}$.

Lors de la consécration de $\mathrm{M}^{\mathrm{gr}}$ Charles Larocque, à Saint-Jean d'Iberville le 29 juillet 1866, les évêques avaient décidé de demander à Rome la création du diocèse de Rimouski ${ }^{52}$. Le $1^{\text {er }}$ mars $1867, \mathrm{M}^{\mathrm{gr}}$ Baillargeon annonçait qu'il avait reçu les bulles pour l'érection du diocèse et la nomination de l'abbé Jean Langevin, alors principal de l'École Normale Laval de Québec, comme premier évêque du nouveau diocèse ${ }^{53}$.

$M^{\mathrm{gr}}$ Taché cherche également à faire diviser son diocèse et à obtenir la nomination de $\mathrm{M}^{\mathrm{gr}}$ Grandin, son coadjuteur, à la tête de ce nouveau territoire qu'il appelle Saskatchewan ${ }^{54} . M^{\text {gr }}$ Faraud, vicaire apostolique d'Athabaska-MacKenzie, s'est fait donner, de son côté, en 1866, un coadjuteur en la personne du Père Clut, o.m.i. Il avait obtenu du Saint-Père la permission de le choisir lui-même sans passer par la filière habituelle. $\mathrm{M}^{\mathrm{gr}}$ Clut sera sacré en août $1867^{55}$.

50 Il était né en Irlande en 1830, était venu au Canada en 1852 . Il fit ensuite ses études de théologie au Séminaire de Montréal. Il devait donc parler le français. D'après The New Catholic Encyclopedia (New York, 1967); voir aussi Baillargeon à Lynch, 5 janvier 1867, AAQ RL 28: 667.

51 Baillargeon à Cooke, 11 septembre 1865, AAQ, RL 28: 390.

52 Baillargeon à Taché, 29 janvier 1867, AAQ, RL 28: 700 .

53 Baillargeon aux Evêques, $1^{\text {er }}$ mars 1867, AAQ RL 28: 713. Il est aussi question entre les évêques de créer un vicariat apostolique pour tous les Indiens du nord, entre la Baie James et le Labrador. On veut le confier aux Oblats. Le supérieur provincial hésite, croit qu'il vaudrait mieux faire deux vicariats, l'un à l'est et l'autre à l'ouest. La Propagande renvoie la question au quatrième concile qui doit se tenir en mai 1868, Guigues à Bourget, 9 mai 1867, ACAM, 255.110, 867-3; Baillargeon à Larocque, 15 novembre 1867, AAQ, RL 29: 105; G. Carrière, Histoire documentaire ... (Ottawa, 1957-), 9: 56-63; Baillargeon à Guigues, 8 février 1868, AAQ, RL 29: 139.

54 Taché au Pape, 26 juin 1867, APFR, SOGG (1868) 1203. M8r Grandin ne deviendra évêque titulaire qu'en septembre 1871, lors de la création de la province ecclésiastique de Saint-Boniface. Voir Don Paul Benoit, Vie de Mor Taché (Montréal, 1904), 2: 144.

55 Faraud à Bourget, 23 novembre 1867, ACAM, 255.115, 867-1. 
Mentionnons enfin par ailleurs que $\mathrm{M}^{\mathrm{gr}}$ Turgeon meurt le 26 août 1867 , après plus de douze ans de maladie. Son coadjuteur-administrateur, $\mathrm{M}^{\mathrm{gr}}$ Baillargeon, devenait par le fait même archevêque de Québec ${ }^{56}$.

L'année 1867 aura donc amener de nombreux changements dans le corps épiscopal de la province ecclésiastique. C'est sans aucune relation avec les transformations politiques qui s'opèrent alors par la Confédération. La province ecclésiastique continue simplement à se développer et à se structurer par le seul fait de l'expansion démographique, de l'augmentation des effectifs dans le clergé et par la montée d'une nouvelle génération. Une ère nouvelle s'ouvre cependant pour l'Église canadienne et surtout pour l'Eglise du Québec, mais les évêques n'en prendront conscience que quelques années plus tard.

\section{Quatrième concile, 1868}

$\mathrm{M}^{\mathrm{gr}}$ Baillargeon avait obtenu en 1865 la permission du Saint-Siège de retarder le quatrième concile de Québec, prévu pour 1866. Le délai accordé reportait les assises conciliaires en 1869. Mais lors d'une réunion des évêques, à l'occasion du sacre de $\mathbf{M}^{\mathrm{gr}}$ Walsh à Toronto en novembre 1867, certains prélats avaient fait pression sur l'administrateur de Québec pour qu'il convoquât le concile dès 1868 . Les évêques du Haut-Canada ne voulaient rien de moins que la division de la province ecclésiastique de Québec et la formation d'une nouvelle province ecclésiastique pour le Haut-Canada ${ }^{57}$.

Le concile sera effectivement convoqué par $\mathrm{M}^{\mathrm{gr}}$ Baillargeon pour obéir aux instances de ses collègues. Les évêques demanderont alors la création, non plus d'une mais de deux nouvelles provinces ecclésiastiques. $\mathrm{M}^{\mathrm{gr}}$ Taché, de Saint-Boniface, ne voulait pas être rattaché à Toronto et l'on jugea qu'il valait mieux qu'il devint lui aussi indépendant de Québec ${ }^{58}$.

Les évêques, dans leurs discussions, avaient cependant failli arriver à une impasse parce que $\mathbf{M}^{\mathrm{gr}}$ Guigues, évêque d'Ottawa, voulait absolument demeurer rattaché à la province ecclésiastique de Québec, tandis que les évêques anglophones désiraient inclure le diocèse d'Ottawa dans la nouvelle province ecclésiastique du Haut-Canada. D'autant plus que

56 Baillargeon, Circulaire au clergé, 26 août 1867, MEQ 4: 587.

57 Baillargeon à Larocque, 15 novembre 1867, AAQ, RL 29: 105. Depuis quelque temps déjà, on pouvait déceler une certaine tendance des évêques du Haut-Canada à vouloir régler leurs problèmes entre eux. Maintenant que $\mathbf{M}^{\mathrm{gr}}$ de Charbonnel s'est trouvé un coadjuteur anglophone en la personne de $\mathrm{M}^{\mathrm{gr}}$ Lynch (1859) et a démissionné en faveur de ce dernier (1860), tous les évêques de cette région sont de langue anglaise (si l'on excepte Ottawa). Sans doute aussi que la formation récente de la province civile d'Ontario pousse les évêques du Haut-Canada à réclamer leur indépendance au plan de l'administration ecclésiastique.

58 Taché à Bourget, 29 décembre 1867, ACAM, 255.109; Acta quarti..., 208. 
cette ville était depuis peu la capitale du pays et qu'elle se trouvait appartenir à la province civile d'Ontario. On en vint à un compromis en disant que, dans quelques années, on pourrait diviser le diocèse d'Ottawa en deux partie selon les limites des provinces civiles et qu'alors la section comprise dans la province d'Ontario serait rattachée à la province ecclésiastique du Haut-Canada ${ }^{59}$. Les positions étaient si tranchées que plusieurs évêques écriront à Rome dès le lendemain du concile pour expliquer leur point de vue respectif au Saint-Siège. La Propagande demandera alors de plus amples explications, spécialement sur les possibilités réelles de diviser le diocèse d'Ottawa ${ }^{60}$. Le SaintSiège approuvera enfin la formation de la province ecclésiastique du Haut-Canada sans y adjoindre le diocèse d'Ottawa et sans fixer pour le moment le siège archiépiscopal de cette province ${ }^{61}$. Le siège métropolitain devait être Toronto selon le vơte majoritaire des évêques au quatrième concile. Mais $\mathrm{M}^{\mathrm{gr}}$ Horan, évêque de Kingston, réclamait ce privilège comme titulaire du plus ancien évêché du Haut-Canada. Il avait l'appui de l'archevêque de Québec, mais l'ensemble des autres évêques étaient convaincus que la ville de Toronto avait beaucoup plus d'avenir. C'est ainsi que le successeur de $\mathrm{M}^{\mathrm{gr}}$ de Charbonnel, $\mathrm{M}^{\mathrm{gr}}$ John J. Lynch deviendra le premier archevêque de Toronto en mars $1870^{62}$.

Le projet d'une province ecclésiastique autour de Saint-Boniface était plus complexe encore. La région ne comportait qu'un diocèse et un vicariat: Saint-Boniface et Athabaska-MacKenzie. M $^{\mathrm{gr}}$ Taché avait cependant demandé à Rome, dès avant le quatrième concile de Québec, que son coadjuteur, $\mathrm{M}^{\mathrm{gr}}$ Grandin, devienne évêque titulaire de SaintAlbert en Saskatchewan. $\mathrm{M}^{\mathrm{gr}}$ Grandin craignait, en demeurant coadjuteur de Saint-Boniface, de devenir archevêque lorsqu'il aurait à succéder à $M^{\mathrm{gr}}$ Taché ${ }^{63}$. La Propagande voulut s'assurer que l'on n'allait pas trop vite en demandant la formation d'une province ecclésiastique avec si peu. Cependant, les distances à elles seules justifiaient l'autonomie de Saint-Boniface. Après de nouvelles instances de $\mathbf{M}^{\mathrm{gr}}$ Taché et de $\mathbf{M}^{\mathrm{gr}}$ Grandin, transmises et approuvées par le nouvel archevêque de Québec, $\mathbf{M}^{\mathrm{gr}}$ Taschereau, le Saint-Siège acceptera de considérer la question et la nouvelle province sera créée le 22 septembre $1871^{64}$.

59 Acta Quarti Concilii... (Québec, 1870) 208-209. Voir aussi le procèsverbal de la discussion entre les évêques, AAQ, Conc. pr. 3 ( $4^{\circ}$ concile), congrégation des évêques.

29: 509 .

60 Baillargeon à Bourget, Guigues et Horan, 29 janvier 1870, AAQ, RL

61 Cazeau à Walsh, 17 février 1870, AAQ, RL 29: 465.

62 Lynch, Pastoral Letter, 6 avril 1870, ACAM, 255.104.

63 Grandin à Barnabo, 9 septembre 1870, AFPR, SC (1868) 767; Taschereau à Barnabo, 26 juillet 1871, AAQ, RL 29: 736; Ponenza, août 1871, AFPR, Acta (1871) 415.

64 Don Benort, Vie de Mgr Taché, 2: 144. 
On érigeait en même temps la mission de Saint-Albert en diocèse et $\mathbf{M}^{\mathrm{rr}}$ Grandin en devenait le titulaire. L'on rattachait à cette province le vicariat apostolique de la Colombie britannique. La nouvelle province comprenait donc deux évêchés, Saint-Boniface et Saint-Albert, et deux vicariats Athabaska-MacKenzie et la Colombie.

La province ecclésiastique de Québec se trouvait ainsi réduite aux limites de la province civile de Québec, avec en plus la partie de l'Ontario comprise dans le diocèse d'Ottawa. Après la mort de $\mathbf{M}^{\mathrm{gr}}$ Baillargeon, survenue le 13 octobre 1870, la direction de cette province ecclésiastique devait passer en de nouvelles mains. L'archevêque défunt avait eu le temps cependant de sentir venir la fin et de préparer sa succession. Il avait mis pas écrit les noms des trois candidats qu'il suggérait pour le remplacer ${ }^{65}$.

Or le premier de ces noms était celui de l'abbé Elzéar-Alexandre Taschereau, bien connu comme le défenseur des intérêts de l'Université Laval et comme opposé à l'ultramontanisme intransigeant. $\mathrm{M}^{\mathrm{gr}}$ Bourget, à titre de doyen des évêques suffragants, était chargé de réunir ses collègues pour approuver ou modifier la liste des noms suggérés par $\mathrm{M}^{\mathrm{gr}}$ Baillargeon. Il subit toutes sortes de pressions, de la part de prêtres surtout, pour que le nom de l'abbé Taschereau soit écarté. Il préféra cependant respecter la volonté de l'archevêque défunt ${ }^{66}$. Mais l'évêque de Montréal ne voyait pas venir cette nomination sans appréhension. Aussitôt qu'elle fût connue, vers la mi-février 1871, il demanda à Rome d'accepter sa démission, ou bien de lui donner tout de suite un coadjuteur avec droit de succession, un coadjuteur, disait-il, qui soit capable de continuer la lutte entreprise par lui pour éliminer du pays toute trace de gallicanisme ${ }^{67}$.

Les craintes du premier pasteur de Montréal n'étaient sans doute pas vaines. On verra bientôt $\mathrm{M}^{\mathrm{gr}}$ Taschereau prendre bien en mains les rênes de la province ecclésiastique et entrer en conflit inévitable avec l'évêque de Montréal, qui se sera trouvé entre temps un allié dans le nouvel évêque des Trois-Rivières, $\mathrm{M}^{\mathrm{gr}}$ Laflèche ${ }^{68}$.

Si $\mathrm{M}^{\mathrm{gr}}$ Taschereau demande d'abord que le cinquième concile soit retardé de deux ans et se tienne en mai 1873, ce n'est pas parce qu'il est timoré. Il est sacré archevêque le 19 mars 1871 et le cinquième concile devait s'ouvrir moins de deux mois après. D'ailleurs le concile du Vatican vient de tenir les évêques éloignés de leurs diocèses durant

65 Cazeau à Barnabo, 12 août 1870, AAQ, RL 29: 564.

66 Bourget à Taschereau, 19 mai 1872, ACAM, RLB 20: 46.

67 Bourget à Simeoni, 10 février 1871, ACAM, RLB 19: 332.

$68 \mathrm{M}^{\mathrm{gr}}$ Cooke était mort à Trois-Rivières le 30 avril 1870; $\mathrm{M}^{\mathrm{gr}}$ Laflèche, son coadjuteur, lui succédait alors automatiquement. 
plusieurs mois; ils ne tiennent pas à ce qu'un autre concile soit convoqué immédiatement. $\mathbf{M}^{\mathrm{gr}}$ Taschereau réunit cependant les évêques au lendemain de son sacre, le 20 mars 1871, pour discuter quelques problèmes urgents. Le ton de certains articles du journal Le Nouveau Monde, feuille catholique lancée par $\mathrm{M}^{\mathrm{gr}}$ Bourget en 1868, lui paraît inquiétant. Quelques mois plus tard, c'est Le Journal des Trois-Rivières, organe de l'évêché, qui publie le fameux Programme catholique. On connaît l'origine de ce manifeste. Des avocats de Montréal, associés avec quelques anciens zouaves, voulaient exiger des candidats à l'Assemblée législative ou aux Communes qu'ils s'engagent par avance à défendre les droits de l'Eglise s'ils voulaient avoir l'appui du vote catholique.

$\mathrm{M}^{\mathrm{gr}}$ Taschereau s'oppose à ce programme dès sa parution, donnant comme raison qu'il n'a pas été approuvé auparavant par les évêques. Or, les premiers pasteurs de Montréal et des Trois-Rivières avaient encouragé fortement ses promoteurs. $\mathrm{M}^{\mathrm{gr}}$ Bourget est tellement convaincu que ce serait là le meilleur moyen d'extirper tout reste de gallicanisme et d'étouffer tout développement du libéralisme catholique condamné par le Pape, qu'il s'associe secrètement avec $\mathrm{M}^{\mathrm{gr}}$ Laflèche pour défendre le Programme catholique, même s'il faut faire la guerre aux autres évêques de la province ${ }^{69}$.

La situation devient si tendue que $\mathrm{M}^{\mathrm{gr}}$ Taschereau doit partir pour Rome à la fin de 1872 dans le but de faire modérer ses collègues de Montréal et des Trois-Rivières. Il obtiendra quelques décisions en ce sens. Revenu à Québec au début de 1873 , après trois mois d'absence, il se sentira un peu plus fort pour convoquer le cinquième concile de Québec. Et alors, sans éliminer les questions controversées, il essaiera de réconcilier les tendances les plus opposées à l'intérieur de l'épiscopat.

Mais avec l'année 1873 et le cinquième concile, nous sommes arrivés au terme de notre périple. Il ne nous reste plus qu'à conclure.

\section{CONCLUSION}

Les étapes en histoire sont toujours un peu artificielles mais elles peuvent servir de points de repère à certaines tentatives d'explication. Ainsi je me hasarde à dire que l'année 1873 peut être considérée comme une date importante dans l'histoire religieuse canadienne à plusieurs points de vue. C'est d'abord la première fois que les évêques du Québec

69 Bourget à Lafièche, 6 mai 1871, ACAM, RLB 19: 504. Le désaccord est très profond. Il porte sur la doctrine des relations entre l'Eglise et l'Etat. Peut-être, comme disait $\mathrm{M}^{\mathrm{gr}}$ Laflèche, s'entend-on sur l'affirmation que l'Eglise est supérieure en principe à l'Etat mais on est en désaccord sur les moyens de rendre cette supériorité effective. Les uns veulent partir en croisade et tout conquérir de haute lutte; les autres cherchent plutôt à s'adapter à l'art du possible qu'est la politique. 
vont se retrouver seuls entre eux à un concile de Québec. Cette date marque aussi comme la fin du règne de $M^{\mathrm{gr}}$ Bourget. La démission de ce dernier ne sera acceptée par le Saint-Siège qu'en 1876 mais dès 1871 , et effectivement au retour de Rome de $\mathrm{M}^{\mathrm{gr}}$ Taschereau en 1873, l'évêque de Montréal a pu réaliser qu'il avait en la personne du nouvel archevêque un homme capable de prendre en mains les affaires de la province et de s'opposer à certaines prises de position de ses collègues.

C'est aussi une nouvelle étape qui commence alors pour l'Eglise canadienne, et en particulier pour l'Église du Québec. Quelques année après la Confédération, les évêques se rendent compte qu'il ont gagné quelque chose par l'avènement du nouvel ordre politique. Il leur est beaucoup plus facile désormais de faire appel à un parlement provincial en majorité catholique qu'il ne l'était d'obtenir quelque chose de l'assemblée du Canada-Uni, en majorité protestante. On pourrait presque dire que l'histoire de l'Eglise du Québec commence vraiment à cette époque. Après s'être libérés de la tutelle de l'autorité civile, les évêques tentent les uns d'annexer au profit de l'Église l'autorité politique, les autres, plus modérés, cherchent tout de même à s'assurer la protection du gouvernement, avec tout ce que cette recherche peut comporter à la longue de risques ou même de compromissions.

Jacques GRISÉ, c.s.c., D.E.S. Histoire, Montréal, P.Q. 